\title{
A Conceptual Framework on Defining Businesses Strategy for Artificial Intelligence
}

\author{
Salih Caner \\ Faculty of Business, Girne American University, Turkish Republic of Northern Cyprus \\ E-Mail: salcanc@gmail.com \\ Feyza Bhatti \\ Faculty of Business, Girne American University, Turkish Republic of Northern Cyprus \\ E-Mail: feyzabhatti@gau.edu.tr
}

\begin{abstract}
The technological developments on artificial intelligence (AI) are going to diffuse in all scales of firms in different industries. AI is increasingly used in diverse business functions, including marketing, customer service, cost reduction, and product improvement. Although there exists a large number of studies on AI, those focusing on the businesses are rather rare, and there is no holistic conceptual framework that brings the information on defining AI business strategy. The aim of this paper is to develop a conceptual framework on defining AI business strategy through a systematic literature review (SLR) of research conducted between 2015 and 2019. Consolidating business and technical views of AI, the paper discusses the major elements of AI in business like abilities and limitations of AI, economics and AI, business functions and AI, workforce, industries and $\mathrm{AI}$, and regulations and ethics of $\mathrm{AI}$ on defining $\mathrm{AI}$ business strategy.
\end{abstract}

Keywords: Artificial intelligence, Business strategy, Systematic literature review, Conceptual framework

\section{INTRODUCTION}

Despite the fact that studies related to artificial intelligence (AI) do exist for more than a half-century, the number of studies focusing on the use of AI in businesses only gained momentum over the last five years. The major technology firms have already 
been involved in a competition in AI technologies and applications to improve their processes and thus outcomes.

McCarthy (2007) defines artificial intelligence as the science and engineering of making intelligent machines, especially intelligent computer programs. Intelligence tasks include visual perception, speech recognition, language translation, and making decisions amid uncertainty.

The term "artificial intelligence" is known to be first used in a research paper submitted by John McCarthy and his colleagues in 1955 (McCarthy, Minsky, Rochester, \& Shannon, 1955). Since then, the terms like AI, machine learning, deep learning, cognitive computing, and machine intelligence are used interchangeably to express roughly the same ideas by different experts, which may confuse people who are not familiar with this field. A computer science scholar Pedro Domingos simplifies the relation of these terms by asserting that "AI is the goal; $\mathrm{AI}$ is the planet we're headed to; machine learning is the rocket that's going to get us there. And Big Data is the fuel." (Conick, 2016, p.29). If deep learning should be added to this analogy, it can be asserted that deep learning is the best kind of a rocket available from the family of rockets.

AI studies slowed down in the 1970s till the 1990s because of the inflated hypes and not promising results of its implementations. On the other hand, there have been triumphs of AI, such as the defeat of World Chess Champion Gary Kasparov by IBM's Deep Blue in 1997 and Google's AlphaGo's historic victory against Go legend Lee Sedol in 2016. However, the wide range of AI applications gained a real business value when three significant developments - better algorithms, massive data availability, and more substantial hardware- have facilitated AI to go beyond the human cognitive abilities in visual recognition and natural language processing. For instance, according to AI Index Report 2017, based on the Large Scale Visual Recognition Challenge Competition results, the best AI systems surpassed human visual ability in 2015 . Likewise, the best AI system with an $87 \%$ accuracy rate, which was $60 \%$ in 2015 , beat the human performance holding an $86.8 \%$ accuracy rate on a reading comprehension test (Stanford Question Answering Dataset 2.0) in 2019. These made contemporary times particularly important to investigate what is already known in the area, and therefore this study focuses on academic studies starting from 2015. The articles related to the theory of artificial intelligence in business generally depicts different angles such as technological capability, economics, intelligence dependence of task and jobs, business functions, and application areas of different industries. This study examines different views to better illustrate the impact of $\mathrm{AI}$ in business.

This paper aims to identify and reveal the potential and limitations of AI in a business perspective and to present a holistic conceptual framework on the uses of AI in businesses. In so doing, the paper employs a systematic literature review (SLR) 
approach to investigate and synthesize the studies on the uses and the impact of AI on businesses. The next section details the methodology. This is followed by a presentation of the findings before the implications of the article are discussed in the concluding section.

\section{METHODS}

To implement SLR, the main stages of Tranfield, Denyer, \& Smart (2003) and Gough et al. (2013) have been adopted in this study. These stages are illustrated in Figure 1 .

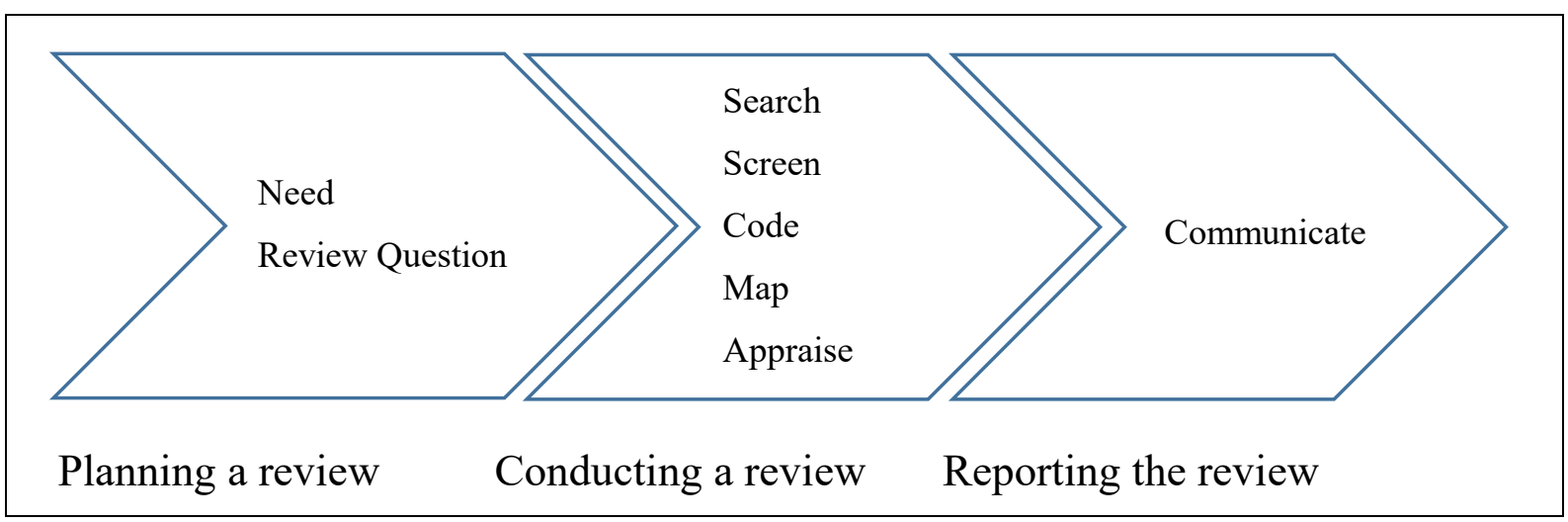

Figure 1 Stages of Systematic Literature Review

\section{Stage 1: Planning a Review}

The need is about explaining the rationale of the research question and clarifying the benefit of resolving the research question. The availability of massive data, better AI algorithms and technologies, and growing computing power have caused significant advancements in AI that lead AI into a higher strategic position for companies and governments. Furthermore, AI is getting more public attention in recent years through broader coverage in the media. The governments are also showing greater attention and inclusion to AI. For instance, the presidents of France and Russia stated the strategic importance of AI for the near future (Gouvernement, 2018; CNBC, 2017), and China declared her ambition to be a leader in AI by 2030 (Shoham, Perrault, Brynjolfsson, \& Clark, 2017). These combine to create the emerging need for a better understanding of AI and its impact on business studies.

Defining the review question describes what the review is trying to achieve. The nature of the problem and available sources determine the review question. Recent breakthroughs such as better algorithms, more substantial hardware, and availability of massive data have made AI technologies to be used in business processes like customer care, marketing, process optimization, human resource allocation, or product innovation. Hence, this study aims to reveal the potentials and limitations of AI from a 
business perspective and to develop a conceptual framework to describe the significant elements of AI. The research questions and the scope of this study are as follows.

What are the different views to explain AI from a business perspective?

What are the impacts of artificial intelligence in businesses?

What are the limitations and risk factors of implementing AI in businesses?

\section{Stage 2: Conducting a Review}

The search strategy defines search parameters and sources which are expected to gather appropriate papers resolving the research question.

The majority of the academic studies about AI are generally published by researchers from technical disciplines like computer science. These valuable studies generally do not provide much insight into the business and management perspective. As of at the end of 2019, a query was executed in the Web of Science academic database for the papers whose topic (abstract, keywords, and title) include "artificial intelligence" string and which are published between 2015 and 2019. As a result, 19,665 articles were found, and $60 \%$ of these studies were from computer science and IT-related disciplines, while $4.2 \%$ percent of studies were either from business, management, economics, or social science interdisciplinary fields. Therefore, inclusion criteria (IC) to select appropriate papers to inspect the impact of AI from a business perspective have been designed as follows.

IC1: Major academic databases were searched like Web of Science, Scopus, Science PG, JStor, Taylor and Francis, Science Direct.

IC2: Publications only from business, management, economics, and social sciences interdisciplinary fields were sought in academic databases.

IC3: The string "Artificial intelligence" was searched in a keyword, title, and abstract of academic papers.

IC4: English academic papers were examined.

IC5: The recently published papers (2015-2019) were selected to obtain up-to-dated knowledge because the majority of the studies in social science concerning AI have been published recently as the business perspective of $\mathrm{AI}$ is an emerging field.

IC6: Google search was performed on the internet to search sources that include both "artificial intelligence" and "business" strings. Mostly, sources of prestigious business consultant firms like Deloitte, McKinsey \& Company, Boston Consulting Group, Accentura, PricewaterhouseCoopers, IBM Institute for Business, and articles of well-known business magazines like Harvard Business Review, MITSloan Management Review, Forbes, and Fortune were reviewed through the internet.

The snowballing technique was also used when the referenced paper was thought 
to have valuable content.

Screening is the process of selecting relevant studies among the ones obtained from the execution of a search query. For example, a query executed in the Web of Science database has returned 839 articles published between 2015 and 2019 in management, business, economy, and social sciences interdisciplinary fields. All titles were assessed, and abstracts of the papers were read to check whether they had valuable insight for further detailed screening.

Theoretical works focusing on the nature of this study, papers related to conceptual frameworks and models specifically concerning to impact of AI in business were selected from the papers returned by the execution of query based on inclusion criterion. If a paper was thought to have a conceptual contribution based on the signal obtained from its abstract and title, then the rest of the paper was inspected. On the other hand, it has been observed that articles obtained according to inclusion criterion have rarely had a conceptual view of business, but they were mainly related to the specific application of AI.

After the potentially relevant studies were selected in the screening phase, useful information was extracted from the particular study taking into account of the research question. In the coding phase, not only the contribution of the study to the research was assessed, but the measures of the quality of the study and characteristics of each study were also highlighted.

Mapping is finding and categorizing specific research features such as the location of the study implemented, the conceptual assumptions involved, characteristics of the samples, the research methods applied, and point of the view of the study relating to the research question. Potential contributing passages of the relevant papers were underlined and prioritized according to the concerns mentioned above. Papers were mapped into six categories based on the views of conceptual frameworks like economics and industries.

The final selection step is to check whether candidate studies conform to the quality and appropriateness measures addressing the review question. Different tools and checklist can be used in SLR to judge for the quality and relevance of the studies. On the other hand, business management research also deals with qualitative studies which are "by nature non-standard, unconfined, and dependent on the subjective experience of both the researcher and the researched" (Tranfield et al., 2003, p.216). Therefore, defining and implementing the quality check step is a major obstacle fulfilling systematic review methodology for management research. In this study, the three general assessment components mentioned by Gough et al. (2013) were used to perform appraising the quality and relevance of studies. The first component is to assess the appropriateness of the study to the research question. Some studies can conform to 
all inclusion criteria, but the overall value of the study may not contribute to the research due to the specificity of that particular study. For example, a social science study related to the credit score prediction system with the help of AI has not been included to review the list because of the specificity of the study. The second component is the measure of the relative contribution of the research design and methods used in a study despite the study was well executed. For example, a study related to the impact of technology in business concerning industry 4.0 has general arguments that do not contribute to this study. The third component is the degree of the exemplary implementation of the study rather than the relevance concerns. Methodology, organization of the paper, the substantiality of supporting arguments, references, and collected data, if any, are the main features of this observation. In this study, the third component is mostly used in the assessment of internet sources.

Synthesis is about connecting the findings and ideas from the studies obtained in prior procedures to answer the research question. Moreover, it is also about revealing insight obtained from papers and directing future research. This process is not only listing findings but integrating ideas to build theories or construct general concepts.

\section{Stage 3: Reporting a Review}

As promising results of the application of AI have been materialized, the power and limitations of AI should be clarified from a business perspective in order to unleash its true potential. By overviewing the studies related to the impact of AI both in academia and business world literature, it has been concluded that there have been different points of view to better explain this phenomenon. These point of views identified as:

Capabilities and limitations of AI

Business Functions and AI,

Economy and AI,

Regulations and AI,

Tasks, Jobs, Intelligence, and AI

Industries and $\mathrm{AI}$

Table 1 depicts the number of articles on each perspective to better define AI in business. The list of articles in Table 1 is shown in the Appendix. 
Table 1 Number of Articles on Each Perspective Constructing AI Business Strategy

\begin{tabular}{|l|c|c|c|}
\hline \multicolumn{1}{|c|}{ Point of Views } & $\begin{array}{c}\text { Number of } \\
\text { Total Articles }\end{array}$ & $\begin{array}{c}\text { Articles Found } \\
\text { in Academic } \\
\text { Journals }\end{array}$ & $\begin{array}{c}\text { Articles Found } \\
\text { in Non-Academic } \\
\text { Sources }\end{array}$ \\
\hline Business Functions and AI & 20 & 6 & 14 \\
\hline Capabilities and Limitations of AI & 15 & 4 & 11 \\
\hline Economy and AI & 9 & 2 & 7 \\
\hline Tasks, Jobs, and AI & 11 & 8 & 3 \\
\hline Industries/Sectors and AI & 17 & 6 & 11 \\
\hline Regulations, Ethics, and AI & 4 & 3 & \\
\hline
\end{tabular}

Note. The list of articles in Table 1 is shown in the Appendix.

Reporting outlines the SLR procedures, presents the sources referenced, and reveals the findings with reasoning. It also needs to discuss the strengths and limitations of the review. However, constructing theories and frameworks in management science based on evidence is not always fully sufficient, clear, and complete. Therefore, Tranfield et al. (2003) preferred to use the term "evidence informed" instead of "evidence based" for the reasoning of findings in SLR in management science.

Because developments in AI have the potential to add economic value in many business areas, studies published in social sciences related to AI have been overviewed to propose a conceptual framework of AI from this perspective. It has been believed that using SLR can help to resolve research questions raised in this study. Therefore, the inclusion criterion of the search strategy has been designed to respond to the concerns mentioned above.

\section{A CONCEPTUAL MODEL OF UTILIZATION OF AI IN BUSINESS}

Most of the academic studies about AI are naturally published in computer science fields, generally dealing with the application of methods and algorithms. As these valuable studies indeed do not contribute much to the conceptual framework of the business perspective, only studies from management related fields were selected for this research. Similarly, most of the studies from social science do not relate to theory building with respect to AI in business. Therefore, non-academic papers published by prestigious business magazines and reports of business consultancy firms were also searched.

While examining the research papers, it was decided that providing insight to exemplify features of a particular industry would help to better understand the impact of AI in business. Therefore, the industrial/sectoral point of view was discussed. However, it is not the intention of this paper to inquire about the effects of AI in different 
industries. The application of AI in marketing has been examined since it can provide insight implementation of AI in many industries.

Because AI-enabled products have automated decision capabilities and $\mathrm{AI}$ is a fast emerging technology, regulation need and addressing ethical questions are critical parts of the successful implementation AI in business. The proposed conceptual framework of critical perspectives of $\mathrm{AI}$ in business is illustrated in Figure 2.

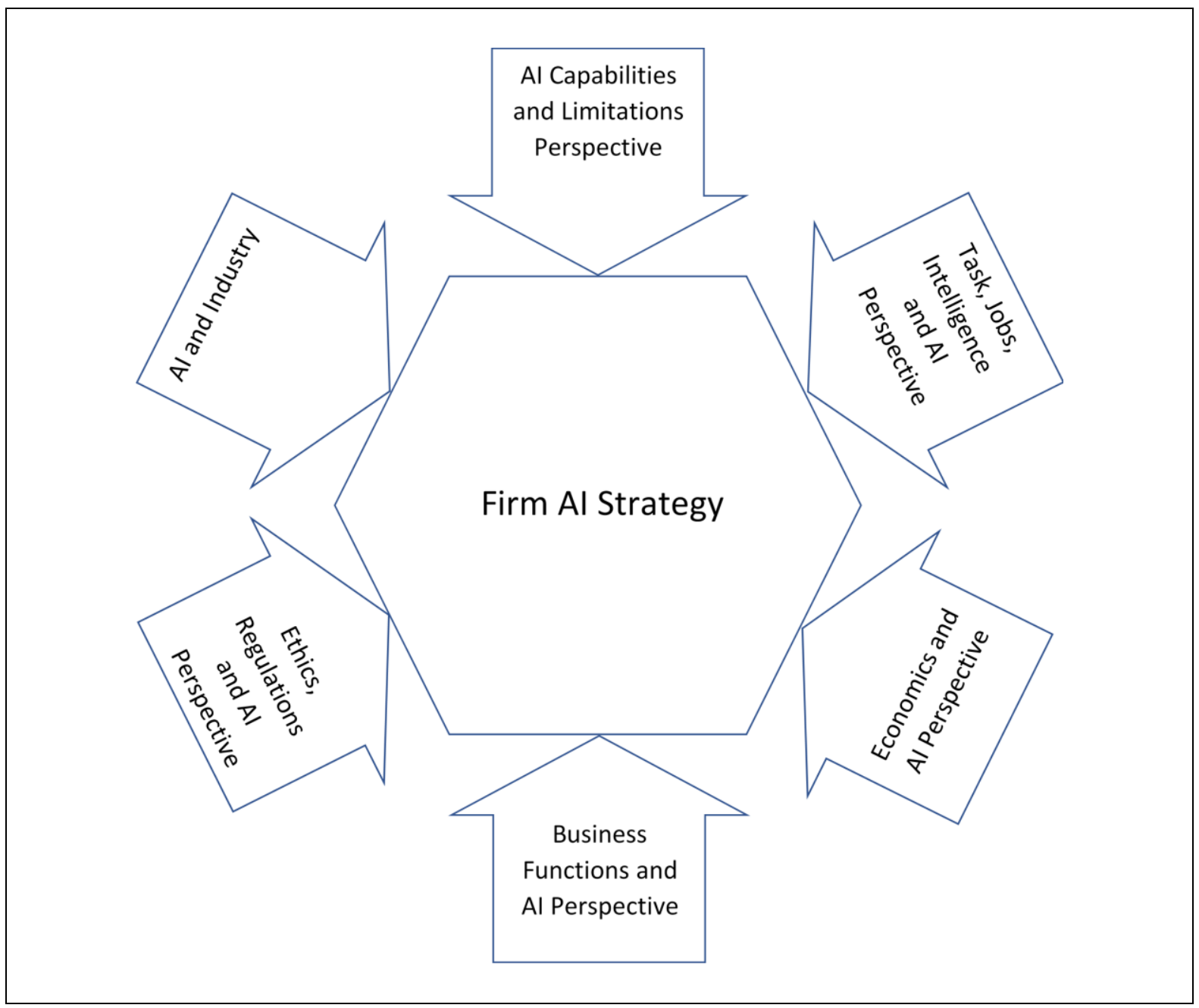

Figure 2 Proposed Conceptual Framework of AI Business Strategy

\section{Capabilities and Limitations of AI}

Machine learning is a subfield of AI that provides computers the ability to learn through the experience without being explicitly programmed (Conick, 2016). There are many machine learning methods such as neural networks, Bayesian network classifiers, decision trees, support vector machines, genetic algorithms etc. One of the famous and successful subfields of machine learning is deep learning which is actually a family of smart algorithms such as deep neural networks and is powered by improvements in computing power and the availability of massive data. 


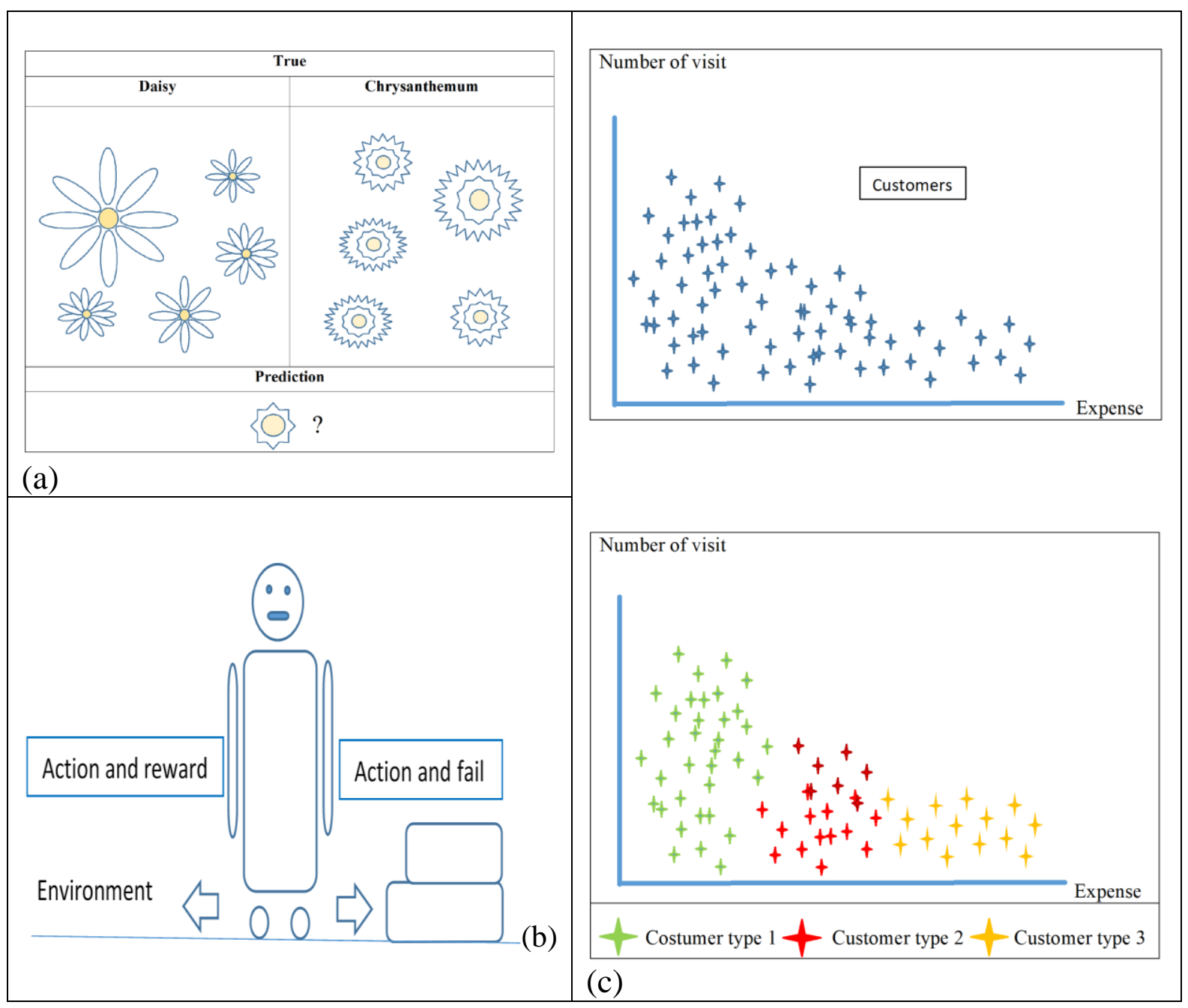

Figure 3 Learning Systems a) Supervised Learning b) Reinforcement Learning c) Unsupervised Learning.

As toddlers learn colors from their parents, computers learn the classification of entities by receiving and analyzing labeled data. Training a learning system with labeled data is called supervised learning, and much of an AI's impact is within this field (LeCun, Bengio, \& Hinton, 2015). For example, by training a vision recognition system with images of daisy and chrysanthemum samples, the system predicts whether a new flower image is a daisy or chrysanthemum (Figure 3). Reinforcement learning (Figure $3 b)$ is a goal-oriented model in such a way that the learning system improves itself by avoiding the decisions that cause failures while selecting decisions that have the maximum possibility of success (Chui, Manyika, \& Miremadi, 2018). This process is similar to learning how to ride a bicycle; one learns by refraining from the actions that cause the rider to fall down. Computer games and robotics which have goals or missionoriented nature use reinforcement learning methods. In unsupervised learning, the AI system receives input data without labels and algorithms search to capture undefined 
structures or clusters within the input space based on differing parameters. The system then categorizes the input space based on their neighborhood characteristics by itself (Jordan \& Mitchell, 2015). The customer segmentation task uses unsupervised learning to uncover new user segments that have not been known before (Figure 3c).

Computer vision, natural language processing, and speech recognition are major AI application domains that have many successful use cases recently. These AI fields also use machine learning techniques in accordance with the specificity of their domains.

Natural Language Processing uses AI, human cognition, and linguistics disciplines to learn, understand, and produce human language content (Hirschberg \& Manning, 2015). Machine to human conversation like Apple's Siri, Amazon's Alexa, Google's Assistant, or customer service chatbots all use NLP. AI can help a human expert to diagnose a disorder by processing health records or to prepare a summary of massive legal documents.

Computer vision is a field of AI that enables computers to process images like humans, such as detecting objects from an image and comprehending the image conceptually. Microsoft announced that their computer vision systems surpassed the human visual cognition ability in 2015 (Eckel, 2015).

There are numerous applications of computer vision, such as online object captioning, diagnosing from MRI or X-ray images, agricultural land monitoring, selfdriving vehicles, optical character recognition, security surveillance, facial detection, robotics etc.

\section{Limitations of AI}

AI applications have the potential to be implemented in many areas, and it is accepted as one of the most disruptive technology in business in the near future. Therefore, its limitations should also be discussed to evaluate the implementation risks. Chui et al. (2018) suggested five AI limitation categories related to business.

Data labeling: Much of the success of AI applications use supervised learning, which requires the labeling input data, which is a time consuming and laborious effort. Big tech companies like Microsoft, Google, Amazon, and IBM offer AI platforms which simplify the AI application building (Garbuio \& Lin, 2018). Additionally, there are alternative methods which produce promising results to lower the burden of labeling effort like reinforcement learning.

Massive training data sets: One of the success factors of the latest AI improvement is the availability of data. While it may not be possible for many companies to obtain data for data-hungry techniques like deep learning, there is no need for every scale of the company to obtain massive data for all the steps of AI 
implementation.

Bias in data and algorithms: AI systems can predict biased decisions because the training data may have hidden biased data. These decisions can cause undesirable circumstances like unfair recruiting decisions, misled scientific or medical prognoses, distorted loan and legal decisions. Resolving biased decisions requires high-level management involvement in data collection and processing steps, application of scrutinized data-science techniques, and human and machine collaboration (Chui et al., 2018).

Generalizability of learning: AI models mostly require different data preparation and training processes for varied use cases since AI has not had human like common sense and inference ability yet. For example, a visual object detection system needs millions of pictures for each different type of vehicle to recognize them efficiently. Computer scientists have been working to tackle this problem. For example, "transfer learning" is a recently developed approach to address this problem.

Explainability: Well-trained AI models mostly have better accuracy than humans in some cognitive areas, but they are unable to explain the reason behind their decisions. The absence of rationality may become an obstacle where reasoning is strictly required. Although, there are emerging approaches to encounter this deficiency like local-interpretable-model-agnostic explanations and generalized additive models (Chui et al., 2018).

The application of AI in business is not limited technologies mentioned above. However, these are the most applied ones in business, such as offering products and services based on customer preferences, deciding process optimization strategy, locating fraud transactions.

AI systems have surpassed the human visual recognition ability in Large Scale Visual Recognition Challenge Competition in 2015 (Shoham et al., 2017). Similarly, AI has beaten the average human score on the Stanford Question Answering Dataset challenge in natural language processing. It is not surprising that as capabilities of AI increase, interest in IA increase in many areas including investment, academia, and public attention.

Implementation of $\mathrm{AI}$ is not confined to the involvement of specific departments in firms. However, the involvement of higher management is needed to define AI investment strategies, as well as data selection approaches and prioritizing and assessing the ethical, security, and privacy concerns. Therefore, business executives' overview knowledge on capabilities and limitations of AI technologies are required to define AI strategy.

\section{Business Functions and AI}


The IBM Institute for Business Value, in cooperation with Oxford Economics, made a survey on 6,050 global executives across 18 industries in order to evaluate the effect of AI on firms. To better evaluate the impact of AI, the most common 13 business functions were categorized into three logical sections: front office, middle office, and back office.

In a front office which consists of departments dealing with customers like sales, marketing, and customer service, AI can cost-effectively increase customer satisfaction and retention rate by more customized approaches, as well as by discovering new consumer segments with the combination of metrics like location, gender, time, and trends. Customer service departments can regulate customer requests by using a combination of AI and conventional approaches to increase satisfaction. Managers can better monitor customer satisfaction both by analyzing current situations and flexibly reorganizing the system.

In the middle office which deals with production issues such as manufacturing, supply chain management, and innovation processes can be optimized for efficiency and productivity. By analyzing data coming from the front office, processes can be regulated depending on customer behavior and other stakeholder's requests by either controlling the supply chain or reforming manufacturing. Managers can make better decisions and generate insight by analyzing AI reports and consulting with staff. AI surveillance systems can act preemptively on critical parts of the manufacturing process. Also, AI has the capability to construct associations among data that may lead to innovations in the future.

In the back office which includes supporting departments like IT, human resources, and finance, AI can automate tasks in order to allow human resources to be allocated to more creative and intuitive tasks. This shift also reduces the percentage of human error on repetitive tasks. For information security concerns, AI has more reliable and faster solutions to detect fraud and discrepancies by processing both structured and unstructured data (Abercrombie, Ezry, Goehring, Marshall, \& Nakayama, 2017).

Davenport and Ronanki (2018) implemented a study on 152 cognitive projects to assess the impact of AI in business. They categorized the effect of AI into three sections: process automation, cognitive insight, and cognitive engagement. According to their study, process automation deals with back-office automation in administrative and financial tasks such as transferring data from e-mail and call center systems into structured database systems and deducing content from legal documents through NLP technologies. Cognitive insight detects patterns from massive amounts of data using machine learning techniques such as predicting purchase behavior, detecting fraudulent activities, and personalized marketing. Cognitive engagement is concerned with automated communication with customers and employees via NLP technologies. 
Applications include 24/7 customer service for most requested services, such as account information changes, answering common employee questions, health disorder diagnoses, and treatment recommendations.

A study implemented by McKinsey Global Institute on 400 AI use cases across 19 industries in nine business functions revealed that marketing \& sales and supplychain management \& manufacturing functions constitute the greatest potential value implementing AI, as it is illustrated in Figure 4 (Chui et al., 2018).

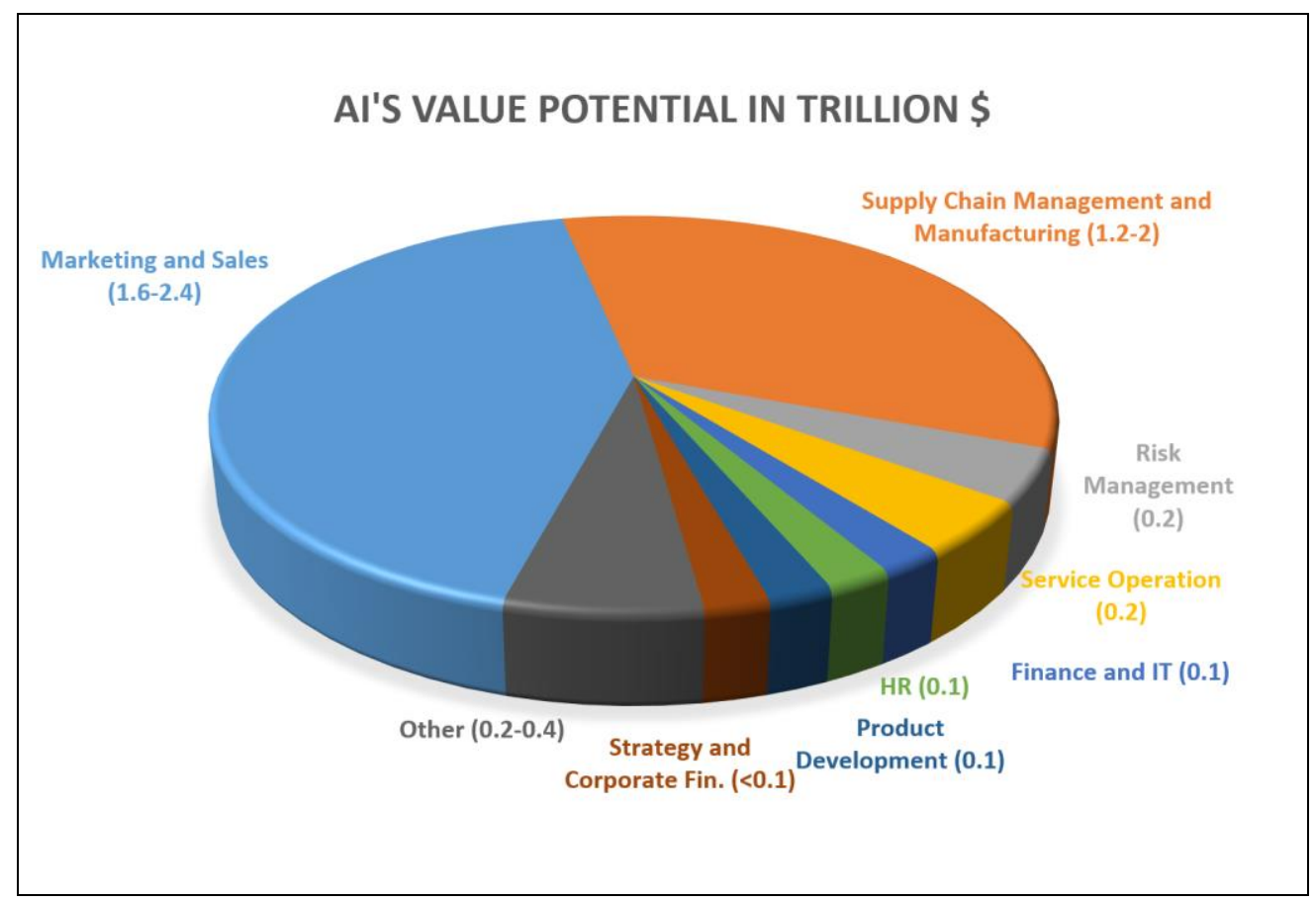

Figure 4 Business Functions Value Potential in Trillion \$ Based on 400 Cases

As information technology has redesigned business functions by changing the implementation of some tasks and facilitating how employees do their jobs, AI has similar potential to change how business functions perform their tasks. AI can practically be used all in business functions. For example, AI can help to design personalized products or services in the production and sales departments. The optimum productivity can be accomplished by the infusion of AI technologies on business functions by reevaluating the tasks and jobs based on the capabilities of AI.

The essence of this perspective is to decide which business functions like production, process management, or marketing that AI can be applied by the collaborative assessment of strategic thinking, available data, and AI capability and nature of tasks of each function.

\section{Tasks, Jobs, and Intelligence}

Rao (2017) suggested that there are three ways that AI can be used in business: 
Assisted intelligence, which is a widely implemented AI that improves current processes carried out in a conventional way. There are a wide variety of other implementations of assisted intelligence, such as customer service chatbots, automated insurance claim processing, and many more.

Augmented intelligence is an emerging field in AI that enables firms to create products, services, or processes that would not be possible without AI. In other words, augmented intelligence is exploring and innovating new business models based on AI. According to Rao (2017), automated machine translation, augmented movie scriptwriting, and legal e-discovery or autonomous legal document processing are the most likely applications of augmented intelligence in the coming years.

Autonomous intelligence, which is expected to be seen in the future, is a fully automatic system that no human involvement is at stake. Self-driving vehicles and automatic stock market trading systems seem to be the early examples of this classification.

Huang and Rust (2018) examined the relation of human intelligence and AI through the history of AI in order to analyze the effect of AI in service. As a result, they have explained the effect of AI with four types of intelligence: mechanical, analytical, intuitive, and empathetic.

Huang and Rust (2018) proposed that AI will replace the tasks based on their intelligence type in a stepwise manner. Firstly, AI will take over routine mechanical tasks. Secondly, it will replace analytical tasks which are complex, data-incentive, and generally predictable. Thirdly, AI will manage to perform intuitive tasks that are creative, complex, experimental, and contextual. Lastly, it will replace empathetic tasks which require managing and influencing other's emotions.

Furthermore, Huang, Rust, and Maksimovic (2019) asserted that there are three levels of AI with respect to intelligence: mechanical, thinking, and feeling intelligence.

Mechanical intelligence is required to perform tasks that are repetitive and mechanical, which need limited learning and adaptation. Mechanical AI has already been well established in business. For example, process optimization facilitated by AI is an instance of this type of intelligence.

Thinking intelligence is about analyzing and evaluating information and making rational decisions. It requires autonomous learning and adaptation based on previous knowledge and current situations. Huang et al. (2019) propose that we all currently experience the thinking economy as thinking AI has well performed the thinking tasks.

As AI has been performing thinking tasks, the human should shift to feeling tasks that require recognizing and responding to human emotions. Huang et al. (2019) have empirically revealed that according to the U.S. government's O*NET occupational data 
in 2006 and 2016 to Feeling Economy is emerging. In other words, human workers need more emotional and communicative skills when AI has been performing thinking tasks.

A study mentioned in McKinsey Global Institute's discussion paper (Bughin et al., 2017) has revealed the same findings with Huang and Rust's theory, as more intuitive and creative jobs have less possibility of automation. The automation possibility of some occupations is given in Table 2, according to the discussion paper.

Table 2 Occupation Types and Their Possibility of Automation

\begin{tabular}{|l|c|}
\hline \multicolumn{1}{|c|}{ Occupation } & Automation Potential \\
\hline Sewing machine operators, agriculture product sorter & $>90 \%$ \\
\hline Stock clerks, travel agents, web developers & $60-80 \%$ \\
\hline Fashion designers, chief executives & $10-30 \%$ \\
\hline Psychiatrist, legislators & $<10 \%$ \\
\hline
\end{tabular}

Generally speaking, the collaboration of AI and humans will produce better results when humans have been shifted tasks requiring more creative, interpersonal, and intuitive skills. Consequently, some jobs and tasks that are repetitive and do not require complex intelligence capacity will be replaced by AI, and the role of humans needs to be redefined. However, for certain tasks, manual monitoring may be necessary in case of ethical concerns or prevent cases that might ruin the prestige of the company. Furthermore, for life-critical missions, AI can only suggest the options to a human expert like medical diagnosis and treatment.

The strategic decisions of this perspective are to consider how to perform collaborative study between humans and AI, and redefine tasks and jobs. By matching the capabilities of AI and the conventional way of performing tasks, AI can help to increase productivity. For example, by initiating an AI enabled chatbot in the customer service department to respond to basic requests, call center employees can deal with complex customer requests that chatbot may not enable to respond.

\section{Economy and AI}

Economists use basic principles to explain human interactions and behaviors, despite their inherent complexity, and AI is no exception. According to Agrawal et al. (2017), the impact of AI from an economic standpoint is based on the value of prediction. Moreover, AI will not only lower the cost of activities that are predictionbased, but will be a substitute for some other areas where prediction does not play a role. Since AI is utilized more as a low-cost prediction tool, human prediction skills will be used less and less, similar to what digital cameras did to film cameras. On the other 
hand, less demand for human prediction skills will increase the demand for human judgment. In terms of economy, judgment will be complementary to prediction. For example, AI can help human experts make diagnoses, and the treatment plans need human judgment.

Prominent scholars in the field of AI and business, Brynjolfsson and Mitchell (2017) asserted that six non-technological factors explain the impact of AI on wages and the workforce.

Substitution: AI will replace some tasks that are conventionally performed by humans and reduce labor demand.

Price elasticity: AI may reduce the cost of some human workforce tasks that can lead to a reduction or increase of total spending, depending on the elasticity of demand.

Complementarities: When AI automates task A which has a complementary task $\mathrm{B}$, it is expected that the price of task A reduces. As the price of task A decreases, demand for task B increases. When there is a shortage of task B, the price of task B increases.

Income elasticity: As AI leads more automation, automation may increase the total income of some individuals that may arise the overall spending.

The elasticity of labor supply: When the availability of people is high for a certain type of job or skill, the wages will not increase because of the elasticity of supply. On the other hand, when the availability of people is scarce for a certain type of job or skill, the demand determines the wages of that skill. For example, when there are many candidates for driver positions, wages will not increase.

Business process redesign: Automation and new technologies creates new ways of more efficient production processes either by optimization or revolutionizing. Therefore more consumers can effort to purchase the product that increases demand elasticity.

Research implemented by PricewaterhouseCoopers suggests that AI can raise consumer demand by enabling personalized and/or better quality products or services. According to the report, it is expected at least $10 \%$ increased economic gain in all sectors of the economy by 2030. It is also estimated that AI impacts on labor will not be only creating new jobs, but the unskilled jobs, which constitute $67 \%$ of all jobs will heavily depend on AI (Gillham, Rimmington, Hugh, Verweij, Rao, Roberts, \& Paich, 2018).

The increasing prediction capability of AI over the years is in parallel with the investment in AI startups. According to the annual AI report 2019, AI startup funding has increased with an average annual growth rate of over $48 \%$ between 2010 and 2018 (Perrault et al., 2019).

The core idea of an economical perspective is related to defining the investment 
strategy of AI. For example, future assessment reports on the economic impact of AI and firm's economic performance related to AI can facilitate executive officers to define investment timeline and budget share of AI implementations. In other words, it is related to time and resource allocation planning based on macroeconomic, sectoral and organizational reports and foresight.

\section{Regulations and AI}

AI will allow public agencies to allocate the workforce to more sophisticated tasks by freeing up mundane tasks that are routine and mechanical in nature. The implementation of AI in citizen services mostly fall into five categories: answering questions, filling out and searching documents, routing requests, translation, and drafting documents (Mehr, 2017).

In addition, AI can enable other citizen services such as providing emergency response, enabling custom and low-cost education, detecting fraud and corruption, improving crime reporting, using prediction to target and enact preemptive social services interventions, informing proactive repairs of infrastructure, and anticipating cyberattacks or personal information loss on public websites (Mehr, 2017).

Despite opportunities and wide application areas of AI, there are also risk factors on the implementation of AI. The main dimensions of challenges in implementing AI in the public sector defined by Wirtz, Weyerer, \& Geyer (2018) are as follows:

\section{AI and Law, Regulations, Governance}

-Governance of autonomous intelligence systems: Although AI exceeds human cognition in formulating decisions from a huge amount of data, non-explainable and biased AI decisions are the major risk for AI as a whole. While industries are much focused on the benefits of AI implementation because of its great potential, governments need to fill the gap on the standardization and accountability of AI systems by defining the principles and regulations accordingly.

-Responsibility and accountability: In the same vein of AI governance, the responsibility of AI systems should also be defined in case of harmful results of AI decision making. For example, who should be blamed if an autonomous public transportation system is involved in an incident in which a pedestrian is injured?

-Privacy/Safety: AI systems should concern for protecting people's privacy and protecting data. For example, a teenager's father argued a department store manager for sending baby clothing coupons and cribs ads to his daughter. In fact, it turned out that his daughter was really pregnant as the AI system had predicted the case by previous purchase behaviors of the customers (Hill, 2012). 


\section{AI Ethics}

It refers to the challenge of AI systems to act and make decisions ethically. This includes a wide range of moral codes, social norms, moral beliefs and behaviors.

-AI rulemaking for human behavior concerns about AI-based decision-making that negatively affects humans. For example, an AI system may reject an application based on biased data.

-Compatibility of the machine and human judgment is about the difference between machine-based decision making and humane values, which may not be adequately reflected in AI systems.

-Moral dilemmas refer to situations in which AI systems need to choose between conflicting alternatives. For example, when an autonomous vehicle is faced with two harmful options, how should the AI system decide?

-AI discrimination is the potential for biased data that cause discriminative decisions.

\section{AI Society}

-Workforce substitution and transformation refers to the impact of AI on the labor market. As it was explained in the previous sections, routine and mechanical tasks will gradually be replaced by AI. However, AI will lead to more jobs that combine human and machine intelligence.

-Social acceptance/trust in AI refers to the fact that there is a great concern for losing control over AI, dealing with ethical dilemmas, and the negative impact on the human workforce. Therefore, these concerns should be addressed concisely in order to gain social acceptance.

The essence of the decision-making mechanism of AI depends on data collected from various sources such as customers, transactions, sensors, devices, etc. However, biased data that might result in undesirable consequences based on automated decisions. Similarly, in some cases, regulatory standards are needed to materialize the AI-enabled product. If the regulatory and robustness concerns were not carefully managed, the reputation of firms would be ruined because of the adverse effect of the product or service. For example, an Independent High Level Expert Group on AI set up by European Commission has recently published "Ethics of Guidelines For Trustworthy AI" to promote lawful, ethical, and robust AI implementation (Independent High-Level Expert Group on Artificial Intelligence, 2019).

An ethical and regulatory perspective is one of the crucial elements of business AI strategy that might ruin the prestige of the company if it has not been handled properly. For example, Google has apologized to a black man who acclaimed that Google's photo algorithms categorized his black friend's photo under the "gorillas" label (Guynn, 
2015). Therefore, ethical and regulatory concerns should be handled at the executive level to refrain a broad range of side effects that might occur.

\section{Industries and AI}

AI has the potential to be used in many sectors like healthcare, automobile, energy, mining, finance, marketing, agriculture, security, IT, transportation, retail and ecommerce, education, and insurance. To better explain AI's impact from the sectoral perspective, the use of AI in marketing has been briefly explained not because its impact is stronger but to exemplify the impact of AI from a specific industry.

\section{Marketing}

A survey on 3500 marketing leaders implemented by Salesforce revealed that at least $60 \%$ of marketing leaders around the world believed AI would change the way of performing digital marketing in the coming five years (Salesforce Research, 2017).

Recommendation engines provide offerings with the help of AI algorithms based on similar preferences or transaction features. They are well-known and the most applied marketing technology used by e-Commerce sites. This technology is so critical that the competitive advantage of Amazon's book or product recommendations, Spotify's ability to discover music taste, and Netflix's movie recommendation precision are the core of their success (Portugal, Alencar, and Cowan, 2018).

By offering much more targeted ads, marketers spend less time on conventional ad techniques. By matching peoples who are most likely to buy their products using historical data, marketers gain a much higher return on investment.

Programmatic advertising uses AI to autonomously buy the ad space in real-time based on the consumer's specific demographics and search behaviors. Instead of implementing a marketing search where and who is your customer, AI-powered bidding system decides which ad to show up in an ad space of a web site based on a visitor's online behaviors like search keys and links clicked (Peterson, 2018).

By processing customer's online footprints, AI can assess customer loyalty and propensity to churn. When an AI system predicts a tendency of losing a customer, customer service can preemptively act to retain the customer.

One of the latest studies regarding the economics of AI estimates that AI will have a substantial impact across all sectors within 5 to 10 years (Gillham et al., 2018). The aim of this study is not to reveal different aspects of AI in the entire industry, but to point out the fact that the impact of AI is not limited to technology firms, but also covers a wide range of applications across different sectors.

\section{DISCUSSION AND FUTURE STUDIES}

Three driving forces of AI, which are better algorithms, stronger computing power, and massive data, have made AI technologies to predict more accurately provide an 
opportunity for firms to have a competitive advantage if the right AI strategy has been constructed. As AI systems have become more precise, AI technologies have attracted more firms. As more AI-enabled products and services such as AI assistants, selfdriving vehicles, or autonomous credit application qualification system have been materialized, new regulatory or ethical problems have aroused. Therefore, governments and regulatory authorities need to become into the stage for the successful implementation of $\mathrm{AI}$ in business. Consequently, there are different concerns and views that have to be considered for the right AI strategy for firms. By reviewing theoretical articles related to AI in business, authors have asserted that six major elements are decisive factors on defining AI strategy for firms as they have been elaborated in the previous sections.

For the perspective of the framework and different propositions, quantitative studies can be asserted in the future to measure the effects of these elements.

After the dark periods, AI technology seems to provide a competitive advantage for firms if their strengths and weakness have been well understood.

Proposition 1: Elaboration of the capabilities, necessities, and limitations of AI throughout the firm provides successful AI implementation.

The AI strategy must be well-coordinated between AI technology and concepts of each business function, including a selection of data, defining the relation between task concepts with technology, and fine-tuning the AI system.

Proposition 2: AI teams from different functions within the organization will provide a better AI strategy for firms.

Solely relying on the capabilities of AI may result in unintended consequences because of biased data or uncovered ethical situations. While AI takes the place of humans in some tasks, human monitoring and guidance role may improve AI implementation.

Proposition 3: Collaborative use of AI and the human workforce has a positive impact on firm performance.

As AI has been taking over new areas in different sectors and employees leave some tasks to AI, more ethical concerns will arise because of the automated decisions. 
Proposition 4: As more AI-enabled products have been used, regulatory and ethical adjustments are needed for more successful AI implementations.

\section{CONCLUSION}

There is more news about the development of new AI applications because of the recent improvements in AI technologies. AI is not a magical tool that will outsmart humans neither a hype that will fade away, but it has the potential to change how we do things. Its capacity, necessities, and limitations should be well introduced at the executive level to construct the right AI strategy.

The main purpose of this article was to reveal what factors might affect constructing AI strategy in business by reviewing theoretical articles on this matter. To reach this goal, the authors performed a systematic literature review to construct a broad theory to explain how to set AI business strategy. Three major steps were followed in the systematic review, namely, planning the review, conducting the review, and reporting the review that was all presented in detail in the previous sections. Consequently, six points of view have been asserted to better explain the appropriate AI strategy in business.

AI helps to better serve customers, create an innovative product, allocate employees to more creative tasks, and reduce costs by implementing in different organization structures (AI and business functions). AI technology has surpassed human cognitive ability in some key areas, such as image processing and natural language processing. There are limitations of AI such biased predictions and unexplainability of autonomous decisions (AI and capabilities and limitations). AI has not been equipped yet with the range of some cognitive capabilities of humans. Therefore, AI can be used at tasks that are routine, voluminous, and not requiring intuitive intelligence. There are risks of extinction of some tasks and jobs and ethical problems in the execution of AI applications. Matching AI capabilities with the nature of the tasks and jobs should be evaluated through the organization structure (AI, tasks, and jobs). AI technologies should be implemented in close scrutiny since automated decisions could ruin a firm reputation if ethical and regulatory cases were not well operated (AI and regulations). AI technology can be used in a wide variety of industries. For example, AI has been revolutionizing the marketing industry by enabling a personalized approach to customers. The latest researches reveal that all sectors will be substantially impacted by AI within ten years (AI and industries). It is not a fallacious argument asserting that AI is a destructive technology that we will observe its substantial impact across all sectors within near future. However, strategic time and resource allocation planning are needed based on macroeconomic and competitive foresight (AI and economy). 
AI is not a technological tool that is confined to technology departments in firms, but it is a tool that depends on the analysis of proprietary data coming from customers, transactions, or processes. Therefore, a collaborative approach of technical and managerial skills is needed for the successful implementation of AI. The aim of this study to reveal the different perspectives of AI, namely capabilities and limitations of AI, regulatory, economic, business functions, tasks and intelligence, and application of different industries. Therefore, the author believes that the conceptual overview of AI from the management science perspective might help to better define AI strategy in firms. In addition, this study may help to define the right questions to assess the effect of $\mathrm{AI}$ in business for quantitative studies.

\section{REFERENCES}

Abercrombie, C., Ezry, R., Goehring, B., Marshall, A., \& Nakayama, H. (2017, June). Accelerating enterprise reinvention: How to build a cognitive organization. The IBM Institute for Business Value. https://www-01.ibm.com/common/ssi/cgibin/ssialias?htmlfid=GBE03838USEN\&

Agrawal, A., Gans, J. S., \& Goldfarb, A. (2017, February 7). What to expect from artificial intelligence. MIT Sloan Management Review.

https://sloanreview.mit.edu/article/ what-to-expect-from-artificial-intelligence/

Brynjolfsson, E., \& Mitchell, T. (2017). What can machine learning do? Workforce implications. Science. 358(6370), 1530-1534.

https://doi.org/10.1126/science.aap8062

Chui, M., Manyika, J., \& Miremadi, M. (2018, January). What AI can and can't do (yet) for your business. McKinsey Quarterly.

https://www.mckinsey.com/business-functions/ mckinsey-analytics/ourinsights/what-ai-can-and-cant-do-yet-for-your-business

CNBC (2017, September 4). Putin: Leader in artificial intelligence will rule world. CNBC. https://www.cnbc.com/2017/09/04/putin-leader-in-artificial-intelligencewill-rule-world.html

Conick, H. (2016, January). The past, present and future of AI in marketing. American Marketing Association.

https://www.ama.org/publications/MarketingNews/Pages/past-present-future-aimarketing.aspx

Davenport, T. H., \& Ronanki, R. (2018, January). Artificial intelligence for the real world. Harvard Business Review, 108-116.

https://hbr.org/2018/01/artificial-intelligence-for-the-real-world

Eckel R. (2015, February 10). Microsoft researchers' algorithm sets ImageNet challenge milestone. Microsoft Research Blog. 
https://www.microsoft.com/en-us/research/blog/microsoft-researchers-algorithmsets-imagenet-challenge-milestone/

Garbuio, M., \& Lin, N. (2018). Artificial intelligence as a growth engine for health care startups: Emerging business models. California Management Review, 61(2), 5983. https://doi.org/10.1177/0008125618811931

Gillham, J., Rimmington, L., Hugh, D., Verweij, G., Rao, A., Roberts, K. B., Paich, M. (2018). Macroeconomic impact of AI. PricewaterhouseCoopers.

Gough, D., Oliver, S., \& Thomas, J. (2013). Learning from research: Systematic reviews for informing policy decisions: A quick guide. A paper for the Alliance for Useful Evidence. Nesta.

Gouvernement (2018, March 30). Artificial intelligence: "Making France a leader".

Gouvernement. https://www.gouvernement.fr/en/artificial-intelligence-makingfrance-a-leader

Guynn, J. (2015, July 1). Google photos labeled black people' gorillas'. Usa Today. https://www.usatoday.com/story/tech/2015/07/01/google-apologizes-afterphotos-identify-black-people-as-gorillas/29567465/

Hill, K. (2012, February 16). How target figured out a teen girl was pregnant before her father did. Forbes. https://www.forbes.com/sites/kashmirhill/ 2012/02/16/how-target-figured-out-a-teen-girl-was-pregnant-before-herfather-did/\#67b818f56668

Hirschberg, J., \& Manning, C. D. (2015). Advances in natural language processing. Science, 349(6245), 261-266.

https://doi.org/10.1126/science.aaa8685

Huang, M., \& Rust, R. T. (2018). Artificial intelligence in service. Journal of Service Research, 21(2), 155-172.

https://doi.org/10.1177/1094670517752459

Huang, M., Rust, R., \& Maksimovic, V. (2019). The feeling economy: Managing in the next generation of artificial intelligence (AI). California Management Review, 61(4), 43-65.

https://doi.org/10.1177/0008125619863436

European Commission. (2019, April 9). Ethics guidelines for trustworthy AI.

European Commission. https://ec.europa.eu/digital-single-market/en/news/ethicsguidelines -trustworthy-ai

Jordan, M. I., \& Mitchell, T. M. (2015). Machine learning: Trends, perspectives, and prospects. Science 349(6245), 255-260. https://doi.org/10.1126/science.aaa8415

LeCun, Y., Bengio, Y., \& Hinton, G. (2015). Deep learning. Nature, 521(7553), 436444. https://doi.org/10.1038/nature14539 
McCarthy, J., Minsky, M. L., Rochester, N., \& Shannon, C. E. (1955). A proposal for the Dartmouth summer research project on artificial intelligence. Computer Science Department. Stanford University.

http://www-formal.stanford.edu/jmc/history/dartmouth/dartmouth.html

McCarthy, J. (2007, November 12). What is Artificial Intelligence? Computer Science Department. Stanford University.

http://www-formal.stanford.edu/jmc/whatisai.pdf

Bughin, J., Hazan, E., Ramaswamy, S., Chui, M., Allas, T., Dahlström, P., Henke, N., \& Trench, M. (2017). Artificial intelligence: The next digital frontier?. McKinsey Global Institute. https:/www.mckinsey.com/ /media/McKinsey/ Industries/Advanced\%20Electronics/Our\%20Insights/How\%20artificial\%20in telligence $\% 20 \mathrm{can} \% 20 \mathrm{deliver} \% 20 \mathrm{real} \% 20$ value $\% 20$ to $\% 20$ companies/MGIArtificial-Intelligence-Discussion-paper.ashx

Mehr, H. (2017). Artificial intelligence for citizen services and government. Ash Center for Democratic Governance and Innovation, Harvard Kennedy School. https://ash.harvard.edu/files/ash/files/artificial_intelligence for_citizen_services. pdf

Perrault, R., Shoham, Y., Brynjolfsson, E., Clark, J., Etchemendy, J., Grosz, B., Lyons, T., Manyika, J., Mishra, S., \& Niebles, J. C. (2019). The AI index 2019 annual report. AI Index Steering Committee, Human-Centered AI Institute, Stanford University, Stanford.

https://hai.stanford.edu/sites/default/files/ai_index_2019_report.pdf

Peterson, A. (2018, August 24). How artificial intelligence is used in targeted marketing. Azati. https://azati.com/artificial-intelligence-targeted-marketing/

Portugal, I., Alencar, P., \& Cowan, D. (2018). The use of machine learning algorithms in recommender systems: A systematic review. Expert Systems with Applications, 97, 205-227. https://doi.org/10.1016/j.eswa.2017.12.020

Rao, A. (2017, May 10). A strategist's guide to artificial intelligence.

Strategy+business. https://www.strategy-business.com/article/A-Strategists-

Guide-to-Artificial-Intelligence?gko=0abb5

Salesforce Research (2017). Fourth Annual State of Marketing.

https://www.salesforce.com/content/dam/web/en_us/www/assets/pdf/datasheets/s alesforce-research-fourth-annual-state-of-marketing.pdf

Shoham, Y., Perrault, R., Brynjolfsson, E., \& Clark, J. (2017). AI index annual report. Stanford's Human-Centered AI Institute. https://aiindex.org/2017/

Tranfield, D., Denyer, D., \& Smart, P. (2003). Towards a methodology for developing evidence-informed management knowledge by means of systematic review.

British Journal of Management, 14(3), 207-222. 
https://doi.org/10.1111/1467-8551.00375

Wirtz, B.W., Weyerer, J. C., \& Geyer C. (2018). Artificial intelligence and the public sector-Applications and challenges. International Journal of Public Administration, 42(7), 596-615. https://doi.org/10.1080/01900692.2018.1498103

\section{APPEDIX The List of Article In Table 1}

\section{Business Functions}

1. Brynjolfsson, E., \& McAfee, A. (2017, July 26). The business of artificial intelligence. Harvard Business Review. https://hbr.org/cover-story/2017/07/the-business-of-artificialintelligence

2. NZ Business Management (2016, October.). How to develop an effective emerging technologies strategy. NZ Business + Management.

3. Abercrombie, C., Ezry, R., Goehring, B., Marshall, A., Nakayama, H. (2017). Accelerating enterprise reinvention: How to build a cognitive organization. The IBM Institute for Business Value.

4. Bataller, C., \& Harris, J. (2016). Turning artificial intelligence into business value. Today. https://pdfs.semanticscholar.org/a710/a8d529bce6bdf75ba589f42721777bf54d3b.pdf

5. Brock, J. K. U., \& Von Wangenheim, F. (2019). Demystifying AI: What digital transformation leaders can teach you about realistic artificial intelligence. California Management Review, 61(4), 110-134.

6. Chui, M., Manyika, J., Miremadi, M., Henke, N., Chung, R., Nel, P., \& Malhotra, S. (2018). Notes from the AI frontier: Insights from hundreds of use cases. McKinsey Global Institute. https://www.mckinsey.com/ /media/mckinsey/featured\%20insights/artificial\%20intelli gence/notes $\% 20$ from $\% 20$ the $\% 20$ ai $\% 20$ frontier $\% 20$ applications $\% 20$ and $\% 20$ value $\% 20 \mathrm{o}$ f\%20deep \%20learning/notes-from-the-ai-frontier-insights-from-hundreds-of-use-casesdiscussion-paper.ashx

7. Davenport, T. H., \& Ronanki, R. (2018). Artificial intelligence for the real world. Harvard business review, 96(1), 108-116.

8. Dawar, N., \& Bendle, N. (2018). Marketing in the age of Alexa. Harvard Business Review, 96(3), 80-86.

9. Finlay, S. (2017). Artificial intelligence and machine learning for business. A NoNonsense Guide to Data Driven Technologies.

10. Jarrahi, M. H. (2018). Artificial intelligence and the future of work: Human-AI symbiosis in organizational decision making. Business Horizons, 61(4), 577-586.

11. Butner, K., Lubowe, D., Ho, G. (2017). The human-machine interchange: How intelligent automation is reconstructing business operations. IBM Institute of Value Chain.

12. Koehler, J. (2018). Business process innovation with artificial intelligence: Levering benefits and controlling operational risks. European Business \& Management, 4(2), 5566. 
13. Lauterbach, A., Bonime-Blanc, A. (2016). Artificial intelligence: A strategic business and governance imperative. NACD Directorship, September/October, 54-57.

14. Makridakis S. (2017). The forthcoming artificial intelligence (AI) revolution: Its impact on society and firms. Futures, 90, 46-60.

15. Chui, M. (2017). Artificial intelligence the next digital frontier?. McKinsey and Company Global Institute, 47, 3-6.

16. Moncrief, W. C. (2017). Are sales as we know it dying . . . or merely transforming?. Journal of Personal Selling \& Sales Management, 37(4), 271-279.

17. Preece, A. (2018). Asking 'Why'in AI: Explainability of intelligent systemsperspectives and challenges. Intelligent Systems in Accounting, Finance and Management, 25(2), 63-72.

18. Ransbotham, S., Kiron, D., Gerbert, P., \& Reeves, M. (2017, Sep. 6). Reshaping business with artificial intelligence: Closing the gap between ambition and action. MIT Sloan Management Review, 59(1). https://sloanreview.mit.edu/projects/reshapingbusiness-with-artificial-intelligence/

19. Ransbotham, S., Gerbert, P., Reeves, M., Kiron, D., \& Spira, M. (2018, Sep. 17). Artificial intelligence in business gets real. MIT Sloan Management Review and Boston Consulting Group. https://sloanreview.mit.edu/projects/artificial-intelligencein-business-gets-real/

20. Schatsky, D., Muraskin, C., \& Gurumurthy, R. (2015). Cognitive technologies: The real opportunities for business. Deloitte Review, 16, 115-129.

\section{Capabilities and Limitations}

1. Ng, A. (2016, November. 9). What artificial intelligence can and can't do right now. Harvard Business Review. https://hbr.org/2016/11/what-artificial-intelligence-canand-cant-do-right-now

2. Timmermans, B. (2017, December 11). Machine Learning Overview. Benjamin Timmermans. https://btimmermans.com/2017/12/11/machine-learning-overview/

3. Brynjolfsson, E., \& Mcafee, A. N. D. R. E. W. (2017). The business of artificial intelligence. Harvard Business Review, 1-20. https://hbr.org/cover-story/2017/07/thebusiness-of-artificial-intelligence

4. Chui, M., Manyika, J., Miremadi, M., Henke, N., Chung, R., Nel, P., \& Malhotra, S. (2018). Notes from the AI frontier: Insights from hundreds of use cases. McKinsey Global Institute.

https://www.mckinsey.com/ /media/mckinsey/featured\%20insights/artificial\%20intell igence/notes $\% 20$ from $\% 20$ the $\% 20$ ai $\% 20$ frontier $\% 20$ applications $\% 20$ and $\% 20$ value $\% 2$ 0of\%20deep\%20learning/notes-from-the-ai-frontier-insights-from-hundreds-of-usecases-discussion-paper.ashx

5. Harper, J. (2017, April 29). The future is now: Cognitive computing throughout the enterprise today. KM World 2017. https://neurons.ai/blog/news-stories/the-future-isnowcognitive-computing-throughout-the-enterprise-today/

6. Jordan, M. I., \& Mitchell, T. M. (2015). Machine learning: Trends, perspectives, and prospects. Science, 349(6245), 255-260. 
7. Hirschberg, J., \& Manning, C. D. (2015). Advances in natural language processing. Science, 349(6245), 261-266.

8. Louis Columbus (2018). 10 charts that will change your perspective on artificial intelligence's growth. Forbes.

https://www.forbes.com/sites/louiscolumbus/2018/01/12/10-charts-that-will-changeyour-perspective-on-artificial-intelligences-growth/\#5096b6f34758

9. Makridakis, S. (2017). The forthcoming Artificial Intelligence (AI) revolution: Its impact on society and firms. Futures, 90, 46-60.

10. Sehgal, M. (2018). Lecture notes from artificial intelligence is the new electricity by Andrew Ng. Medium. https://manavsehgal.com/lecture-notes-from-artificialintelligence-is-the-new-electricity-by-andrew-ng-4712dcbf26e5

11. Bughin, J., Hazan, E., Ramaswamy, S., Chui, M., Allas, T., Dahlström, P., Henke, N., $\&$ Trench, M. (2017). Artificial intelligence: The next digital frontier?. McKinsey Global Institute. https://www.mckinsey.com/ /media/McKinsey/Industries/ Advanced\%20Electronics/Our\%20Insights/How\%20artificial\%20intelligence\%20can \%20deliver\%20real\%20value\%20to\%20companies/MGI-Artificial-IntelligenceDiscussion-paper.ashx.

12. Heath, N. (2018). What is AI? Everything you need to know about artificial intelligence. Managing AI and ML in the Enterprise, ZDNet.

https://www.zdnet.com/article/what-is-ai-everything-you-need-to-know-aboutartificial-intelligence/

13. Purdy M., \& Daugherty P. (2016). Why artificial intelligence is the future of growth. Accenture. https://www.accenture.com/t20170524T055435 w /ca-en/_acnmedia/ PDF-52/Accenture-Why-AI-is-the-Future-of-Growth.pdf

14. Parloff, R. (2016). From 2016: Why deep learning suddenly changing your life. Fortune.com. https://fortune.com/longform/ai-artificial-intelligence-deep-machinelearning/

15. Syam, N., \& Sharma, A. (2018). Waiting for a sales renaissance in the fourth industrial revolution: Machine learning and artificial intelligence in sales research and practice. Industrial Marketing Management, 69, 135-146.

\section{Economy}

1. Agrawal, A., Gans, J., \& Goldfarb, A. (2018). Prediction machines: The simple economics of artificial intelligence. Harvard Business Press.

2. Agrawal, A., Gans, J., \& Goldfarb, A. (2016). The simple economics of machine intelligence. Harvard Business Review, 17, 2-5.

3. Agrawal, A., Gans, J., \& Goldfarb, A. (2017). What to expect from artificial intelligence. MIT Sloan Management Review. https://sloanreview.mit.edu/article/whatto-expect-from-artificial-intelligence/

4. Brynjolfsson, E., \& Mitchell, T. (2017). What can machine learning do? Workforce implications. Science, 358(6370), 1530-1534.

5. Bughin, J., Hazan, E., Ramaswamy, S., Chui, M., Allas, T., Dahlström, P., Henke, N., \& Trench, M. (2017). Artificial intelligence: The next digital frontier?. McKinsey Global Institute. https://www.mckinsey.com/ /media/McKinsey/Industries/ 
Advanced\%20Electronics/Our\%20Insights/How\%20artificial\%20intelligence\%20can\% 20deliver\%20real\%20value\%20to\%20companies/MGI-Artificial-IntelligenceDiscussion-paper.ashx.

6. Agrawal, A., Gans, J., \& Goldfarb, A. (2018, Apr. 24,). The economics of artificial intelligence. McKinsey Quarterly. https://www.mckinsey.com/businessfunctions/mckinsey-analytics/our-insights/the-economics-of-artificial-intelligence\#

7. Gillham, J., Rimmington, L., Hugh, D., Verweij, G., Rao, A., Roberts, K.B., Paich M. (2018). The macroeconomic impact of AI. PricewaterhouseCoopers.

8. Szczepański, M. (2019). Economic impacts of artificial. European Parliamentary Research Service.

9. Perrault, R., Shoham, Y., Brynjolfsson, E., Clark, J., Etchemendy, J., Grosz, B., Lyons, T., Manyika, J., Mishra, S., \& Niebles, J. C. (2019). The AI index 2019 annual report. AI Index Steering Committee, Human-Centered AI Institute, Stanford University, Stanford, CA. https://hai.stanford.edu/sites/default/files/ai_index_2019_report.pdf

\section{Intelligence}

1. Rao, A. (2017). A strategist's guide to artificial intelligence. Strategy + business. https://www.strategy-business.com/article/A-Strategists-Guide-to-ArtificialIntelligence?gko=0abb5

2. Brougham, D., \& Haar, J. (2018). Smart technology, artificial intelligence, robotics, and algorithms (STARA): Employees' perceptions of our future workplace. Journal of Management \& Organization, 24(2), 239-257.

3. Brougham, D., \& Haar, J. (2018). Smart technology, artificial intelligence, robotics, and algorithms (STARA): Employees' perceptions of our future workplace. Journal of Management \& Organization, 24(2), 239-257.

4. Chui, M., Manyika, J., Miremadi, M., Henke, N., Chung, R., Nel, P., \& Malhotra, S. (2018). Notes from the AI frontier: Insights from hundreds of use cases. McKinsey Global Institute. https://www.mckinsey.com/ /media/mckinsey/featured\%20insights/ artificial\%20intelligence/notes\%20from\%20the\%20ai\%20frontier\%20applications \%2 0and $\% 20$ value $\% 20 \mathrm{of} \% 20 \mathrm{deep} \% 20$ learning/notes-from-the-ai-frontier-insights-fromhundreds-of-use-cases-discussion-paper.ashx

5. DeCanio, S. J. (2016). Robots and humans-complements or substitutes?. Journal of Macroeconomics, 49, 280-291.

6. Ferràs-Hernández, X. (2018). The future of management in a world of electronic brains. Journal of Management Inquiry, 27(2), 260-263.

7. Jarrahi, M. H. (2018). Artificial intelligence and the future of work: Human-AI symbiosis in organizational decision making. Business Horizons, 61(4), 577-586.

8. Huang, M. H., \& Rust, R. T. (2018). Artificial intelligence in service. Journal of Service Research, 21(2), 155-172.

9. Bughin, J., Hazan, E., Ramaswamy, S., Chui, M., Allas, T., Dahlström, P., Henke, N., \& Trench, M. (2017). Artificial intelligence: The next digital frontier?. McKinsey Global Institute. https://www.mckinsey.com/ /media/McKinsey/Industries/ Advanced\%20Electronics/Our\%20Insights/How\%20artificial\%20intelligence\%20can 
\%20deliver\%20real\%20value\%20to\%20companies/MGI-Artificial-IntelligenceDiscussion-paper.ashx

10. Kaplan, A., \& Haenlein, M. (2019). Siri, Siri, in my hand: Who's the fairest in the land? On the interpretations, illustrations, and implications of artificial intelligence. Business Horizons, 62(1), 15-25.

11. Wright, S. A., \& Schultz, A. E. (2018). The rising tide of artificial intelligence and business automation: Developing an ethical framework. Business Horizons, 61(6), 823-832.

\section{Ethical}

1. Wirtz, B. W., Weyerer, J. C., \& Geyer, C. (2019). Artificial intelligence and the public sector-applications and challenges. International Journal of Public Administration, 42(7), 596-615.

2. Mehr, H., Ash, H., \& Fellow, D. (2017). Artificial intelligence for citizen services and government. Ash Center for Democratic Governance and Innovation, Harvard Kennedy School.

https://ash.harvard.edu/files/ash/files/artificial_intelligence_for_citizen_services.pdf

3. Independent High-Level Expert Group on Artificial Intelligence. (2019). Ethics guidelines for trustworthy AI. Eurepan Commission. https://ec.europa.eu/futurium/en/ai-alliance-consultation

4. Wright, S. A., \& Schultz, A. E. (2018). The rising tide of artificial intelligence and business automation: Developing an ethical framework. Business Horizons, 61(6), 823-832.

\section{Sectoral}

1. Chui, M., Manyika, J., Miremadi, M., Henke, N., Chung, R., Nel, P., \& Malhotra, S. (2018). Notes from the AI frontier: Insights from hundreds of use cases. McKinsey Global Institute. https://www.mckinsey.com/ /media/mckinsey/featured\%20insights/ artificial\%20intelligence/notes $\% 20$ from $\% 20$ the $\% 20$ ai $\% 20$ frontier $\% 20$ applications $\% 2$ 0and $\% 20$ value $\% 20$ of $\% 20$ deep $\% 20$ learning/notes-from-the-ai-frontier-insights-fromhundreds-of-use-cases-discussion-paper.ashx

2. Desouza, K. C. (2018). Delivering artificial intelligence in government: Challenges and opportunities. IBM Center for the Business of Government.

3. Faggella, D. (2018). Artificial Intelligence in Marketing and Advertising - 5 Examples of Real Traction. Techemergence.

4. Garbuio, M., \& Lin, N. (2018). Artificial intelligence as a growth engine for health care startups: Emerging business models. California Management Review, 61(2), 5983. https://doi.org/10.1177/0008125618811931

5. Conick, H. (2016). The past, present and future of AI in marketing. American Marketing Association. https://www.ama.org/publications/MarketingNews/ Pages/past-present-future-ai-marketing.aspx

6. Kaplan, A., \& Haenlein, M. (2019). Siri, Siri, in my hand: Who's the fairest in the land? On the interpretations, illustrations, and implications of artificial intelligence. Business Horizons, 62(1), 15-25. 
7. Kietzmann, J., Paschen, J., \& Treen, E. (2018). Artificial intelligence in advertising: How marketers can leverage artificial intelligence along the consumer journey. Journal of Advertising Research, 58(3), 263-267.

8. Winans, M. (2017). Cognitive insights will revolutionize how marketers deepen customer connections and fuel strategic growth. White paper. IBM Marketing Cloud. ftp://ftp.www.ibm.com/software/in/pdf/10_Key_Marketing_Trends_for_2017.pdf

9. Marr B. (2018). How is AI used in healthcare - 5 powerful real-world examples that show the latest advances. Forbes.

10. Bughin, J., Hazan, E., Ramaswamy, S., Chui, M., Allas, T., Dahlström, P., Henke, N., \& Trench, M. (2017). Artificial intelligence: The next digital frontier?. McKinsey Global Institute. https://www.mckinsey.com/ /media/McKinsey/Industries/ Advanced\%20Electronics/Our\%20Insights/How\%20artificial\%20intelligence\%20can \%20deliver\%20real\%20value\%20to\%20companies/MGI-Artificial-IntelligenceDiscussion-paper.ashx.

11. Peterson, A. (2018, August 24). How artificial intelligence is used in targeted marketing. Azati Software. https://azati.com/artificial-intelligence-targeted-marketing/

12. Popomaronis, T. (2017). 11 Tech leaders share the real truth about artificial intelligence (and what really matters). Forbes. https://www.forbes.com/sites/tompopomaronis/2017/08/29/11-tech-leaders-shareinsights-on-artificial-intelligence-and-what-actually-matters/\#f64d1cb66687

13. Portugal, I., Alencar, P., \& Cowan, D. (2018). The use of machine learning algorithms in recommender systems: A systematic review. Expert Systems with Applications, 97, 205-227.

14. Parloff, R. (2016). From 2016: Why Deep learning is suddenly changing your life. Fortune.com. https://fortune.com/longform/ai-artificial-intelligence-deep-machinelearning/

15. Salesforce Research (2017). Fourth Annual: State of Marketing. Salesforce Research. https://c1.sfdcstatic.com/content/dam/web/en_us/www/assets/pdf/datasheets/salesforc e-research-fourth-annual-state-of-marketing.pdf

16. Syam, N., \& Sharma, A. (2018). Waiting for a sales renaissance in the fourth industrial revolution: Machine learning and artificial intelligence in sales research and practice. Industrial Marketing Management, 69, 135-146.

17. West, A., Clifford, J., \& Atkinson, D. (2018). "Alexa, build me a brand" An Investigation into the impact of Artificial Intelligence on Branding. The Business \& Management Review, 9(3), 321-330.

Mr. Salih Caner (Corresponding author) is currently a $\mathrm{PhD}$ candidate at the Faculty of Business and Economics of the Girne American University in Girne, Turkish Republic of Northern Cyprus. He has received his BA in Computer Science Engineering from Hacettepe University, and MS in Informatics Institute of Middle East Technical University, Ankara. His MS study was related to applications of pattern recognition techniques on medical data. His research interest is on artificial intelligence and robotics from business management perspective. 
Dr. Feyza Bhatti is the Vice Dean and an assistant professor of Faculty of Business and Economics at the Girne American University, Turkish Republic of Northern Cyprus. She is also the Director of the Center for Gender Studies and Vice Director of Graduate Studies and Research at the same University. She received her BA in Economics from Bilkent University, Ankara, and MS in Economics from Eastern Mediterranean University, and thereafter her PhD degree in Sociology from University of Edinburgh. She is a mixed-methods social science researcher with substantive experience and interest in gender and development, sociology of family, labor economics, and qualitative management research. 